\title{
Early post-traumatic pulmonary-embolism in patients requiring ICU admission: more complicated than we think!
}

\author{
Mabrouk Bahloul ${ }^{1}$, Mariem Dlela ${ }^{1}$, Nadia Khlaf Bouaziz ${ }^{2}$, Olfa Turki ${ }^{1}$, Hedi Chelly ${ }^{1}$, Mounir Bouaziz ${ }^{1}$ \\ ${ }^{1}$ Department of Intensive Care, Habib Bourguiba University Hospital, Sfax, Tunisia; ${ }^{2}$ Centre Intermédiaire, Rte El MATAR Km 4, Sfax, Tunisia \\ Correspondence to: Professor Mabrouk Bahloul. Department of Intensive Care, Habib Bourguiba University Hospital, 3029 Sfax, Tunisia. \\ Email: bahloulmab@yahoo.fr.
}

Submitted Jun 10, 2018. Accepted for publication Sep 12, 2018.

doi: $10.21037 /$ jtd.2018.09.49

View this article at: http://dx.doi.org/10.21037/jtd.2018.09.49

We read with interest the article entitled "Prevalence and main determinants of early post-traumatic thromboembolism in patients requiring ICU admission" (1). In this retrospective study, Kazemi Darabadi et al. (1) had included to their database, the records of 240 trauma-patients requiring ICU admission, with a confirmed diagnosis of pulmonary embolism (PE). The patients were categorized as subjects with an early PE ( $\leq 3$ days) and those with a late PE ( $>3$ days). According to their analysis, $48.5 \%$ of the patients suffering from $\mathrm{PE}$ had developed this complication within $72 \mathrm{~h}$ following a trauma event and/or after ICU admission. This study (1) has confirmed that the patients in early PE group were older than those who suffered late PE $(45.9 \pm 7.49$ vs. $42.6 \pm 8.81$ years; $\mathrm{P}=0.002$ ). In addition, the prevalence rate of long bone fractures in lower extremities was significantly higher in those with early $\mathrm{PE}$ when compared with the other patients $(26.8 \%$ vs. $7.0 \%, \mathrm{P}<0.001)$. Finally, they found that the group with early stage $\mathrm{PE}$ had more severe injuries when compared to those with late $\mathrm{PE}(\mathrm{P}=0.007)$. Using the multivariable logistic regression models, old age (age of the patient), presence of long bone fractures, and a more severe injury could predict occurrence of early $\mathrm{PE}$ in trauma patients when they had been referred to the emergency ward. The conclusion of this study is that the occurrence of early PE can be predicted in a majority of the trauma-patients who were requiring ICU admission especially older patients, patients with long bone fractures and those with severe injury.

Although the incidence of early post-traumatic thromboembolism in trauma-patients requiring ICU admission reported in the literature ranges from $10 \%$ to $30 \%(1-7)$, this is, to the best of our knowledge, the first study that analyzes the main determinants of an early post- traumatic thromboembolism in patients who are requiring an ICU admission. Nevertheless, this study as mentioned by the authors suffers from many limitations. In fact, the small sample size, and also the retrospective design of the study lead to an increased probability of some bias when collecting data. In fact, in this study (1), PE was not screened on a daily basis. As a consequence, the late stage PE may only be a delayed diagnosis. For instance, a patient who develops with PE on day 2 but without any symptom(s); then he is accidentally diagnosed with $\mathrm{PE}$ on day 4 or 5 . One other inherent limitation of a retrospective study is that the diagnostic tool for deep vein thrombosis (DVT) and/or PE can be different for patients. In fact, in some patients the positive diagnosis can be confirmed with ultrasound, while for others, this is done with an enhanced $\mathrm{CT}$ and/or ventilation/perfusion (V/Q) scan.

In addition to these study limitations, many other clarifications and/or limitations have not detailed. First, the prevalence of PE was not studied. Second, it is not clear if patients with traumatic brain injury and/or spine fractures were included in this study. Third, the number of patients requiring a sedative and mechanical ventilation (MV) was not clarified. In addition, Trauma produces from time-dependent responses from the haemostatic system can increase the risk of bleeding of an injury which needs blood transfusion. However, it has been well-established that the cases with hemorrhagic shock and/or those which required blood transfusion are highly associated with the development of an early PE (2). In addition, severe trauma increases the levels of pro-inflammatory procoagulant cytokines, leading to an inflammatory reaction. These points are not analyzed in this study. Furthermore, the 


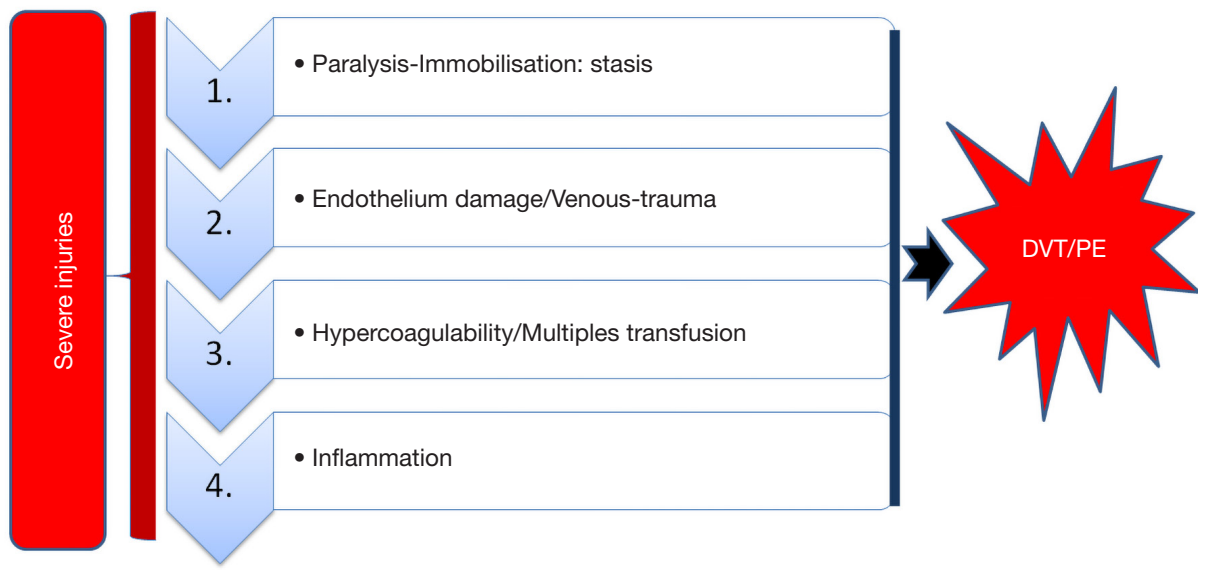

Figure 1 Factors associated to the formation of DVT and PE after trauma. They include risk factors for hypercoagulability, as originally described by Virchow, incorporating the 3 original triad (stasis; endothelial injury; and hypercoagulability) and adding a fourth: inflammation(acute inflammation does increase venous thrombosis). DVT, deep vein thrombosis.

presences of a femoral venous line, operative interventions requiring general anaesthesia, and the use of blood transfusion during the first $72 \mathrm{~h}$, were probably not studied for all patients. Besides, the delay and the type of DVT prophylaxis were not detailed. Moreover, it is not clear why the authors had used a low dose of enoxaparin $(30 \mathrm{mg} / \mathrm{bid})$. Finally, the impact on the outcome of early post-traumatic thromboembolism was not analyzed.

Venous thromboembolism (VTE), which includes a $\mathrm{PE}$ and DVT, remains a major challenge and represents severe complications in the care of critically ill patients (2-4). Indeed, in the ICU, a PE is a difficult diagnosis, which may be missed. This is because of a nonspecific clinical presentation, principally in patients who are requiring $\mathrm{MV}$. The diagnosis of $\mathrm{PE}$ is usually suspected when there is an unexplained hypoxemia and/or shock, and an arterial hypotension occurs. It is well known that computed tomography pulmonary angiography (CTPA) is the gold standard for the diagnosis of PE, showing one or more filling defects or obstruction in the pulmonary artery or its branches. However, a massive PE can cause a cardiac arrest and ultrasound can be helpful in this setting, as CT is not advisable for this setting. In fact, the emergency setting of an extracorporeal cardiopulmonary resuscitation (ECPR) significantly limits the performance of CTPA (8). Thus, echocardiography can be the first modality used in this particular setting. Typical findings of a massive PE include a marked dilation of the right heart, and a compromised left ventricle (LV) (8). Moreover, it is well-established today that severely-injured-patients bear a significantly increased risk for the development of a VTE event, and a PE (2-6). In fact, severe trauma is a hypercoagulable state often complicated with VTE events. Severe trauma increases the levels of the pro-inflammatory procoagulant cytokines (1-3), tissue factor (TF) and markers of a thrombin generation, and increased procoagulant phospholipids and TF-bearing microparticles (1-3,7,9-11). In addition, hypoperfusion and tissue-level injury leads to endothelium damage with a thrombomodulin expression. Moreover, after trauma, the levels of the natural anticoagulants, such as, antithrombin (AT), protein C (PC), and protein $\mathrm{S}(\mathrm{PS})$ are reduced (2) leading to an exposure of VTE complications. We concluded that the factors which are contributing to the formation of DVT and PE after trauma were risk factors for hypercoagulability, as originally described by Virchow, incorporating the 3 original triads: stasis; endothelial injury; and hypercoagulability, as well as adding a fourth, inflammation (12). In Fact, inflammation increases procoagulant factors, and also inhibits the natural anticoagulant pathways and fibrinolytic activity, leading to DVT and/or PE (2) (Figure 1).

Although an effective prophylaxis measurement for thromboembolic disease exists, the most effective ones increase the risk of bleeding $(4,5)$. In fact, given the risk of the aggravation of a bleeding in trauma-patients (in particular with traumatic brain injury), the initiation of pharmacological prophylaxis is commonly delayed for more than 48 hours after ICU admission (3-5). Thus, late initiation may increase the risk of VTE. In fact, an early post-mortem trauma-patient study, which was performed in 1961 by Sevitt and Gallagher (7) has showed an incidence 
of DVT of $65 \%$ and those of PE at $16 \%$, on an autopsy. Despite these findings, the incidence of a thromboembolic event among hospitalized trauma patients varies widely, ranging from less than $1 \%$ to $58 \%$, depending upon the demographics of the study population and the nature of injuries (3-5). The previously reported incidence of posttraumatic PE in the ICU diverges largely, ranging from $0.13 \%$ to $12 \%(3-7)$, because systematic screening has not been performed $(4,6)$. As a consequence, our purpose now in the severe-trauma-patients (example head injury), is to initiate a preventive anticoagulation (with low molecular weight heparin) within 24 hours after ICU admission, if the initial injuries do not worsen on a control brain CT-scan (performed in 24 hours, and at variable intervals thereafter based on clinical manifestations).It is also possible that the preventive dose of an unfractionated heparin $(40 \mathrm{mg}$ of enoxaparin or its equivalent) in our ICU, should be revised up in this specific condition (3-6).

Moreover, recent data has suggested that a significant number of PE occurs very early, and even immediately, after injury $(13,14)$. However, up to now, there has been little focus on the factors associated with the early occurrence of post-traumatic PE. To the best of our knowledge, few studies have identified risk factors predictive of early PE after injury (15-17). On the other hand, they were all retrospective studies, with the inherent limitation of a retrospective study which consists in the fact that the diagnostic tool for DVT and/or PE can be different for patients. In addition, they were all conducted in level 1 trauma centers, and none of them has a concerned ICU population. This study conducted by Kazemi Darabadi et al. (1) is the first study conducted in ICU. Also, in our literature review, we found there to be no uniform definitions of "early" or "late" post-traumatic PE. Usually, early PE was defined as an occurrence of PE $3(1,16-18)$ or 4 days after injury (15).

The study by Brakenridge et al. (15) was the first to elucidate factors which were associated with the timing of a post-traumatic PE (15). Long bone extremity fractures were identified as the only independent risk factor for early PE. On the other hand, late PE groups had a higher injury severity score (ISS), severe head injuries or severe chest injuries, and also a delay in the chemical prophylaxis initiation beyond 24 hours. Similarly, Coleman et al. (16) showed that that for certain injury patterns, such as an extremity abbreviated injury score (AIS) $>3$, were predictive of early PE. Whereas, a severe head injury and spinal cord injury were associated with a higher risk of late $\mathrm{PE}$, as well as, blood transfusions and high ISS. In 2014, Benns et al. (17) reported that early PE patients were more likely to have lower extremity injuries, lower ISS and a shorter average length of stay. In contrast, late PE patients who had suffered from a traumatic brain injury, and had more delay in initiating chemical prophylaxis (17). However, reviewing these studies and on multivariate analysis, there were no differences between the age, sex, body-mass-index, LOS, the use of femoral lines, hypotension, ventilator days nor mortality. Table 1 summarizes the frequency and the majority factors which had been associated with early post-traumatic PE published in the literature. Most recently, Gelbard et al. (18) reported in a retrospective study, that there were no significant differences in the baseline characteristics nor in injury patterns between the early and late PE groups. Yet, they set out to demonstrate that early $\mathrm{PE}$ is not associated with a right ventricular dysfunction when using a computed tomographic measurement, and suggested that early PE may have a different underlying pathophysiology (18). Indeed, Knudson et al. (12), had postulated the ability of a major chest trauma in stimulating inflammation which lead to the direct formation of PE by direct inflammation of pulmonary vessels. This theory may explain why chest injury is associated with $\mathrm{PE}$, but not significantly with DVT (12). In fact, only $20 \%$ of the patients with PE had an associated DVT event (12). We agree with Knudson et al. (12) and their hypothesis. We think that this subject needs further research. In Fact, we agree that the inflammatory process that is initiated by a chest trauma may be forced by a coexisting tissue hypoxia and systemic inflammation (usually associated with severe injury) leading to pulmonary endothelial damages and in situ thrombosis of pulmonary arteries (Figure 2).

We conclude that there were many factors that contributed to the formation of DVT and PE after a trauma injury. In addition to risk factors for hypercoagulability, as originally described by Virchow, incorporating the 3 original triad (stasis; endothelial injury; and hypercoagulability), we suggest in this specific condition, that a fourth factor should be also considered: the role of inflammation is acting via endothelial damage. The role of a major chest trauma in stimulating a local inflammation which leads directly to the formation of $\mathrm{PE}$ by a direct inflammation of the pulmonary vessels should require a further investigation in the future. Prevention is strongly needed. 
Table 1 Summary of included studies

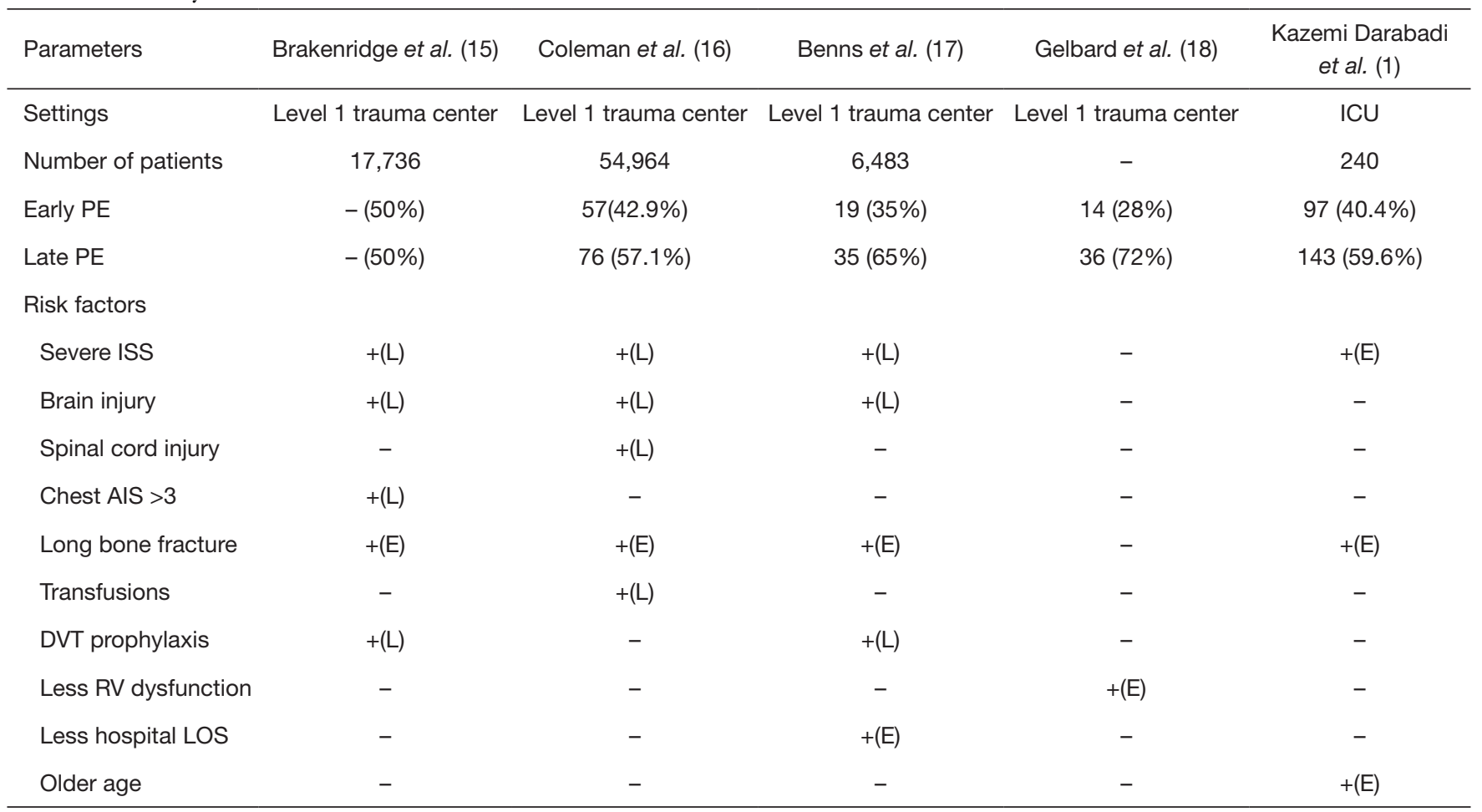

$+(\mathrm{E})$ : in favours early; $+(\mathrm{L})$ : in favours late. PE, pulmonary embolism; AIS, abbreviated injury score; DVT, deep vein thrombosis.

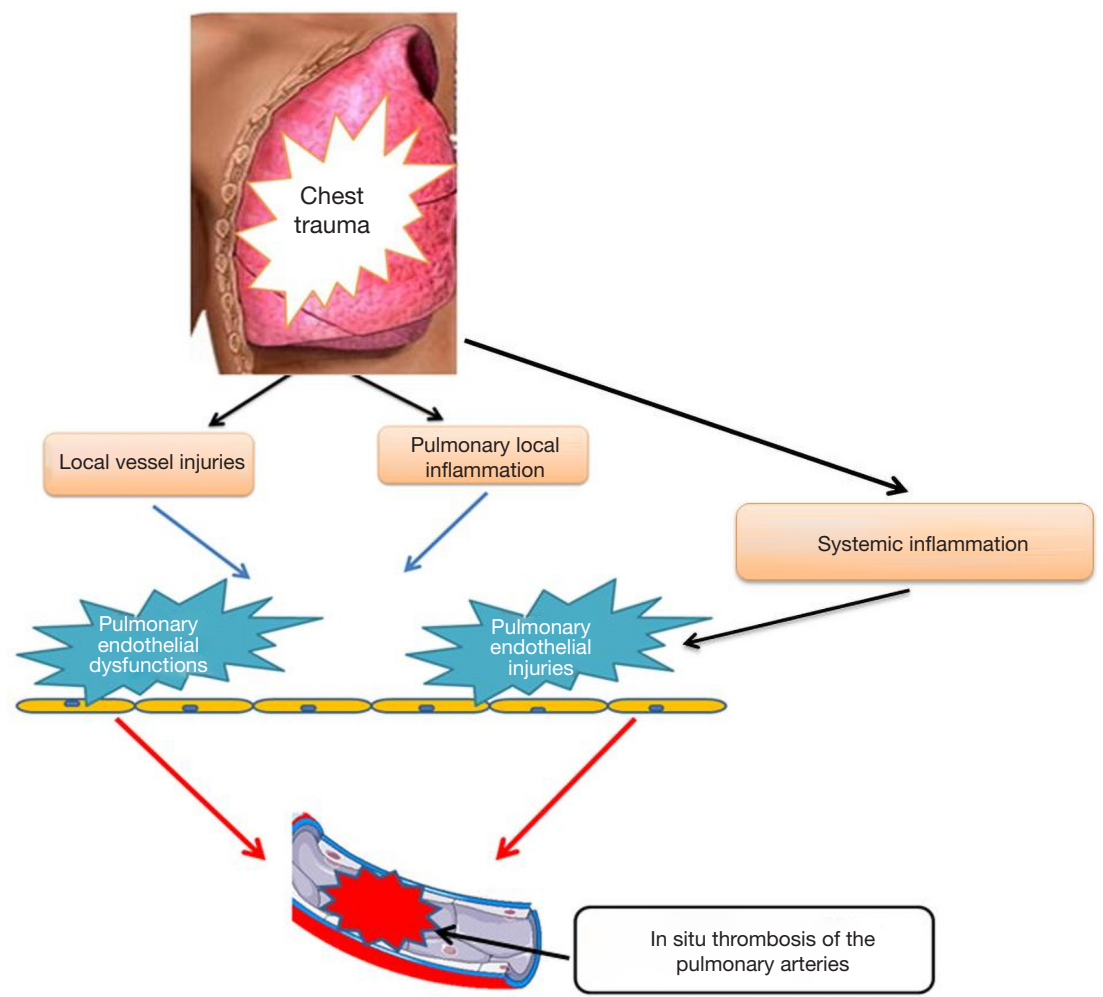

Figure 2 Mechanisms of "in situ thrombosis of pulmonary arteries" in severe chest-trauma patients. 


\section{Acknowledgements}

All authors thank Professor Chokri Khlaf and Miss Nour Bahloul for their help in the redaction of this manuscript.

\section{Footnote}

Conflicts of Interest: The authors have no conflicts of interest to declare.

\section{References}

1. Kazemi Darabadi F, Jafari Zare MA, Torabi Goodarzi Z, et al. Prevalence and main determinants of early posttraumatic thromboembolism in patients requiring ICU admission. Eur J Trauma Emerg Surg 2018;44:133-6.

2. Holley AD, Reade MC. The 'procoagulopathy' of trauma: too much, too late? Curr Opin Crit Care 2013;19:578-86.

3. Bahloul M, Chelly H, Regaieg K, et al. Pulmonary embolism following severe traumatic brain injury: incidence, risk factors and impact outcome. Intensive Care Med 2017;43:1433-5.

4. Bahloul M, Regaieg K, Chtara K, et al. Posttraumatic thromboembolic complications: Incidence, risk factors, pathophysiology and prevention. Ann Cardiol Angeiol (Paris) 2017;66:92-101.

5. Bahloul M, Chaari A, Dammak H, et al. Post-traumatic pulmonary embolism in the intensive care unit. Ann Thorac Med 2011;6:199-206.

6. Skrifvars MB, Bailey M, Presneill J, et al. Venous thromboembolic events in critically ill traumatic brain injury patients. Intensive Care Med 2017;43:419-28.

7. Sevitt S, Gallagher N. Venous thrombosis and pulmonary embolism. A clinico-pathological study in injured and burned patients. Br J Surg 1961;48:475e489.

8. Zhang Z. Echocardiography for patients undergoing extracorporeal cardiopulmonary resuscitation: a primer for

Cite this article as: Bahloul $M$, Dlela $M$, Khlaf Bouaziz N, Turki O, Chelly H, Bouaziz M. Early post-traumatic pulmonary-embolism in patients requiring ICU admission: more complicated than we think! J Thorac Dis 2018;10(Suppl 33):S3850-S3854. doi: 10.21037/jtd.2018.09.49 intensive care physicians. J Intensive Care 2017;5:15.

9. Lichte P, Kobbe P, Almahmoud K, et al. Post-traumatic thrombo-embolic complications in polytrauma patients. Int Orthop 2015;39:947-54.

10. Paffrath T, Wafaisade A, Lefering R, et al. Venous thromboembolism after severe trauma: Incidence, risk factors and outcome. Injury 2010;41:97-101.

11. Cohen AT, Tapson VF, Bergmann JF, et al. Venous thromboembolism risk and prophylaxis in the acute hospital care setting (ENDORSE study): A multinational cross-sectional study. Lancet 2008;371:387-94.

12. Knudson MM, Gomez D, Haas B, et al. Three thousand seven hundred thirty-eight posttraumatic pulmonary emboli: a new look at an old disease. Ann Surg 2011;254:625-32.

13. Spencer Netto F, Tien $\mathrm{H}, \mathrm{Ng}$ J, et al. Pulmonary emboli after blunt trauma: Timing, clinical characteristics and natural history. Injury 2012;43:1502-6.

14. Owings JT. Timing of the Occurrence of Pulmonary Embolism in Trauma Patients. Arch Surg 1997;132:862.

15. Brakenridge SC, Toomay SM, Sheng JL, et al. Predictors of early versus late timing of pulmonary embolus after traumatic injury. Am J Surg 2011;201:209-15.

16. Coleman JJ, Zarzaur BL, Katona CW, et al. Factors Associated with Pulmonary Embolism Within 72 Hours of Admission after Trauma: A Multicenter Study. J Am Coll Surg 2015;220:731-6.

17. Benns M, Reilly P, Kim P. Early pulmonary embolism after injury: A different clinical entity? Injury 2014;45:241-4.

18. Gelbard RB, Karamanos E, Farhoomand A, et al. Immediate post-traumatic pulmonary embolism is not associated with right ventricular dysfunction. Am J Surg 2016;212:769-74.

(English Language Editor: Jeremy Dean Chapnick, AME Publishing Company) 